\title{
Protocol for Measuring Free (Low-stress) Exploration in Rats
}

Wojciech Pisula* and Klaudia Modlinska

Institute of Psychology, Polish Academy of Sciences, Warsaw, Poland

*For correspondence: wojciech.pisula@wp.pl

\begin{abstract}
[Abstract] Research on exploratory behavior plays a key part in behavioral science. Studying exploratory behavior of laboratory rodents may provide important data about many developmental and neurobiological processes occurring in animal ontogenesis. The proposed protocol for measuring the free (low-stress) exploration behavior in rats is straightforward, requires minimal resources and very little animal training. It can therefore be broadly applied to studying animal cognition, animal behavior in general, the aging processes, and several animal models of various phenomena.
\end{abstract}

Keywords: Exploratory activity, Free exploration paradigm, Laboratory rodents, Response to novelty, Habituation

[Background] Research on exploratory behavior is an important part of behavioral science. One of the main goals of this research is to gain a better understanding of the process of adaptation to novelty introduced to a familiar environment or novelty of an unfamiliar environment. Encounters with a novel object or place are crucial for animal survival. They can bring new opportunities or pose a threat. An individual can adapt to the change or try to cope with it in a different way. Specific reactions to novelty depend on the type and characteristics of the novel stimulus and constitute multi-level processes. A high intensity of the stimulus or its biological significance (e.g., food or predator) usually trigger stereotypical responses called 'species-specific defense responses' (SSDR), such as freezing, separating the tail from the body (lizards), burying, spreading putrid odor, or flight (see Fanselow and De Oca, 1998, for an extensive review). Reactions to low-intensity stimuli (e.g., stimuli which are not crucial for survival) are less easily observed. However, they are not less important, since they form the core of behavioral repertoire in many species. One may hypothesize that, since the organism does not deal with this class of stimuli by relying on reflexes, the process seems to occur at a higher organization level, similarly to cognitive or pre-cognitive processes. It is therefore important to understand these behavior regulation mechanisms at various levels of organization, and, consequently, apply methods allowing the researcher to measure behavioral activity in its full complexity. Our protocol, described below, was designed to address the postulate of low-stress testing environment for animals, therefore enabling them to express the whole variety of behavioral repertoire.

Most studies conducted to date involve some form of spatial rearrangement of physical objects or introduction of novel objects in an open field or in the animal's homecage (Calhoun, 1962; Renner and Seltzer, 1991; Picq and Dhenain, 1998; Gouteux et al., 1999). Our protocol replicates this arrangement, but with some notable improvements.

We propose a protocol and equipment suitable for measuring free exploration in small laboratory 
mammals (e.g., rat, mouse, opossum) in conditions ensuring low levels of stress in individuals involved in the experiment. The aim of the proposed protocol is to analyze the new environment exploration, the rate of habituation to it, and the response to the innocuous and low-intensity novelty into a well-known context, in the rat. During the experiment, the animal is allowed to explore the test arena but is not forced to leave the safe area (transporter). What is more, the experimental chamber creates an opportunity to explore various zones both in the horizontal and in the vertical plane.

The proposed equipment and procedure are suitable for various small mammals. However, laboratory rat is the most common species used in exploration studies, due to its high sensitivity to novelty. The initial version of the procedure was applied in a study conducted on RHA/RLA rats (Pisula, 2003) and most recently on Lister Hooded rats (Pisula et al., 2019).

\section{Materials and Reagents}

1. Paper towels and nitrile laboratory gloves

2. Waterproof marking pen

3. Laboratory rats ( 3 months old; weight between 200 and $240 \mathrm{~g}$ ) However, individuals of a different age may be used in studies on developmental or aging processes. The rats are housed in groups of 4 per cage and kept in an environment with controlled temperature $\left(22 \pm 2{ }^{\circ} \mathrm{C}\right)$ and humidity (45-65\%). The animals have a $12-12 \mathrm{~h}$ lightdark cycle and are provided with food and water ad libitum.

4. Virkon ${ }^{\circledR} \mathrm{S}$ (Bayer) (used for cleaning purposes)

Virkon ${ }^{\circledR} S$ is a disinfectant in which the main component $(49.4 \%)$ is a triple salt of potassium: monoperoxy sulphate, potassium hydrogen sulphate and potassium sulphate. It also contains $18 \%$ sodium hexanophosphate, $15 \%$ sodium dodecylbenzenesulphonate, $10 \%$ malic acid and $5 \%$ sulphamic acid.

\section{Equipment}

1. Home cage (Tecniplast ${ }^{\oplus}$ (Italy), 1500 U Eurostandard Type IV)

2. Night vision video camera (BCS-THC3400IR-E, HD-CVI 4MPX IR 30M)

3. Wide angle infrared (IR) illuminator (IR Lab Ltd., model: LIR-CS32-940)

4. Transporter for rats

A device made of opaque material, used to move the animals from the home cage to the test arena (Figure 1). In our experiments we used a custom made transporter constructed with two overlapping hard PVC tubes. Lifting the middle tube (with a string) reveals the entrance cut out in the outer tube. It is used to standardize the procedure and ensure the minimum stress level caused by human handling during the experiment. The transporter should be equipped with a pull-up door/gate. The gate must be left open during the whole trial in order to allow (but not force) the animal to leave the transporter or stay in it. It should be noted however, that any other 
similar solutions may be applied.

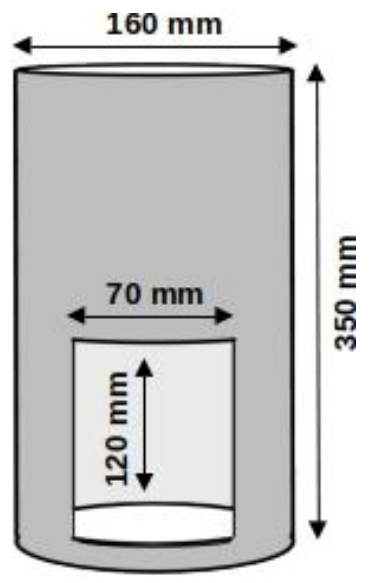

Figure 1. Cylindrical transporter used in our experiment

Note: The exit gate $(70 \mathrm{~mm} \times 120 \mathrm{~mm})$ should match the size of the entrance to the test arena.

5. Chamber for measuring exploratory behavior (Figure 2)

This test arena is a large box (width $=800 \mathrm{~mm}$, height $=800 \mathrm{~mm}$, depth $=600 \mathrm{~mm}$ ). The chamber is divided into three zones ( $A, B$ and $C$ ) which have been separated by two walls running perpendicularly to its longer side. The front and the top of the chamber are made of transparent walls that can be lifted to gain full access to the test arena. The wooden division walls between the zones have triangular entrances $(120 \mathrm{~mm} \times 140 \mathrm{~mm})$ at the bottom, which enables the animals to move freely between the chamber parts. Dividers are placed at an angle, so as to prevent blind spots in the video recording from the top and from the front. The entrance to the chamber is placed in the central zone (A). The remaining zones, $\mathrm{B}$ and $\mathrm{C}$, are where the objects allowing experimental manipulations are placed. In our recent studies (e.g., Pisula et al., 2019), we used wooden tunnels for this purpose (length $=240 \mathrm{~mm}$, width $=120 \mathrm{~mm}$, height $=80 \mathrm{~mm}$ ). The entire chamber is covered with a layer of washable varnish. Wooden tunnels are covered with washable paint.

6. Tunnels (Figure 2)

In contrast to the most frequently used two-dimensional experimental settings, these tunnels provide a complex three-dimensional environment. In our experiments we used tunnels (200 $\mathrm{mm} \times 120 \mathrm{~mm} \times 80 \mathrm{~mm}$ ) made of hardwood covered with washable paint. The tunnels are placed in zones $\mathrm{B}$ and $\mathrm{C}$. The configuration of the tunnels is changed in one of the chamber zones before the first test trial and this change introduces novelty to the experimental plan. Tunnel configuration is changed by manually moving them or adding/subtracting tunnels in two zones of the chambers. 


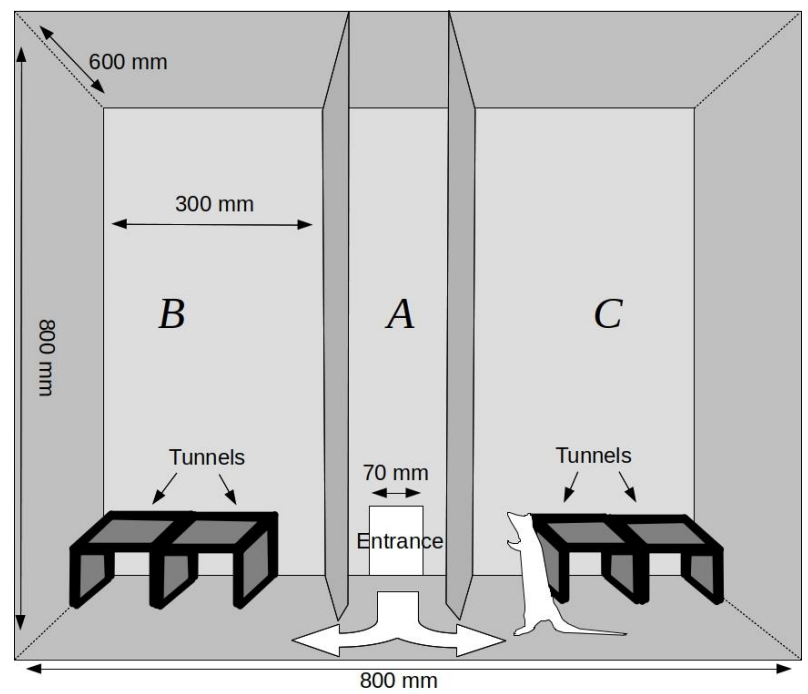

Figure 2. Chamber for measuring free exploration and reaction to low-stress novelty in rats and other small mammals. Frontal view through the transparent front wall.

\section{Software}

1. BORIS event logging software (Friard and Gamba, 2016; http://www.boris.unito.it/), for coding behaviors from recorded materials, or any other software enabling behavior coding on the basis of video recordings

\section{Procedure}

The experiment comprises seven habituation trials followed by three trials involving experimental manipulation (see Videos 1 and 2). Experimental manipulation is achieved by introducing novelty, which takes place between trials 7 and 8 . Three subsequent trials are conducted in the chamber with this new arrangement (see Video 2). Each trial lasts $7 \mathrm{~min}$ and is administered for each animal once a day. 


\section{bĭ́-protocol

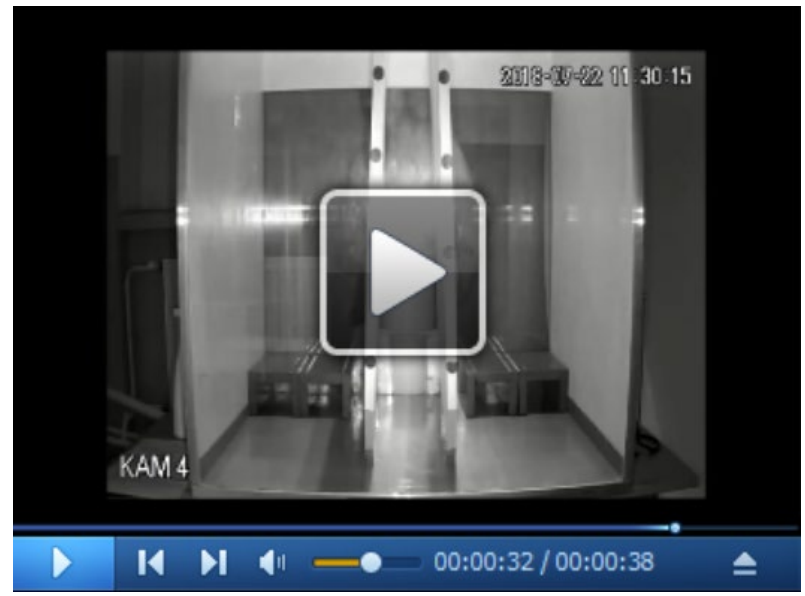

Video 1. Example of habituation trial. (Experimenters are advised to comply with the local ethical requirements regarding animal experimentation.)

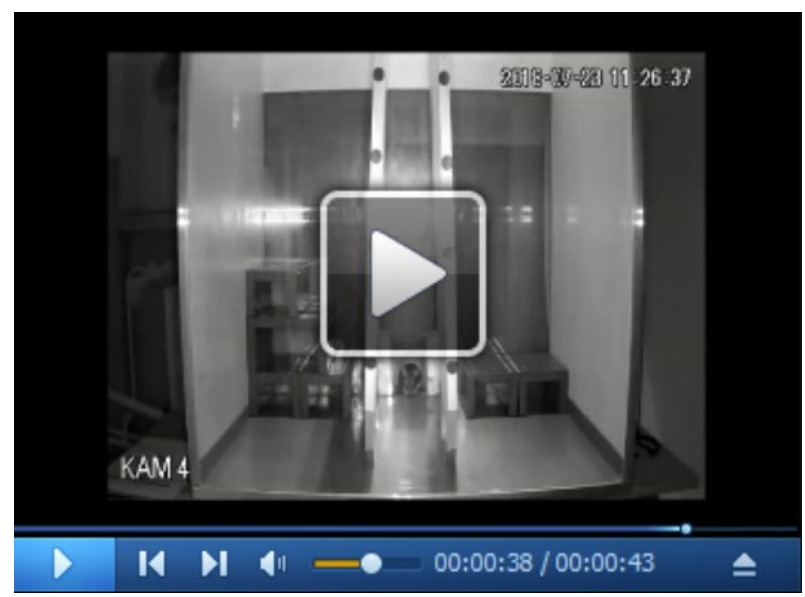

Video 2. Example of test trial. (Experimenters are advised to comply with the local ethical requirements regarding animal experimentation.)

A. Preparation

1. Familiarize the animals with human contact before the experiment.

Note: Since the whole procedure is supposed to ensure the lowest possible stress level, systematic animal handling by the experimenter is strongly recommended. A typical procedure would consist of 3 sessions of 5-15 min per week involving holding, tickling, talking to the animals, etc.

2. Mark the rats' tails with a waterproof marking pen to quickly identify animals in the cage and avoid high stress levels before testing.

3. Arrange the experimental setup as shown in Figure 3. 


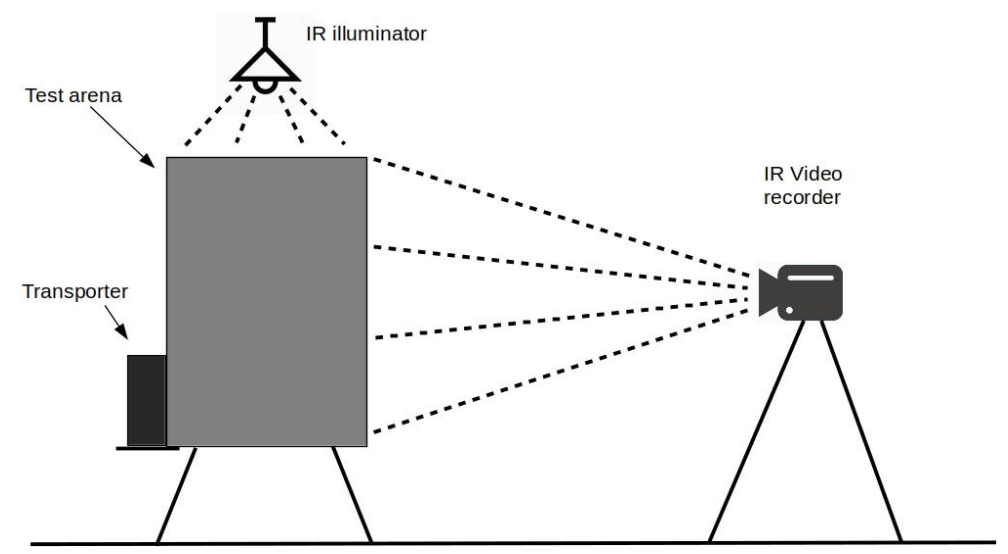

Figure 3. Lateral view of the experimental setting

4. Arrange the object in the experimental chamber in accordance with the experimental protocol (see Figure 4).

\section{Habituation phase (trials 1-7)}

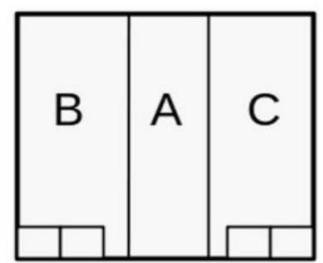

Test phase (trials 8-10)

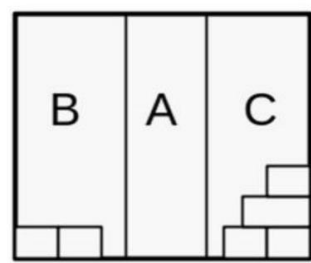

Figure 4. Test arena arrangement during habituation and test trials. An example of a basic experimental plan used previously in Pisula et al., 2019.

5. Reduce background noise to a minimum (alternatively, 'white noise' can be used).

6. Ensure complete darkness in the test arena.

7. Make sure that the whole test arena (both the horizontal and the vertical plane) is clearly visible in VR and the IR lamp is placed correctly and sheds light evenly on the entire horizontal surface through the transparent top cover of the chamber.

8. Make sure that the whole test area has been cleaned with the cleaning agent before the experiment and after each experimental trial (see 'Materials and Reagents' above).

9. Run the tests at the same time each day.

B. Experimental procedure

1. Take the animal out of the home cage and gently place it in the transporter.

2. Lock the transporter.

3. Place the transporter in the initial position attached to the test arena (see Figure 3).

4. Leave the animal undisturbed for 15-20 s for adaptation.

5. Open the transporter gate. Allow the animal to leave the transporter or stay in it. 
Note: The gate should be left open during the whole trial.

6. Leave the experimental room.

7. After a 7-min recording session, enter the experimental room and gently take the animal out of the test arena (it is important not to associate aversive stimulation with any element of the study procedure) and place it in a temporary home cage.

Note: Due to the possible confounding effect of animal interactions, the 'after trial' temporary cage should be placed in another room. This should prevent interaction between the animals waiting for the measurement and the individuals that have already completed the test.

8. Clean the test arena and the transporter thoroughly.

9. Ensure that the objects inside the chamber are placed correctly, that is, in accordance with the experimental protocol for a given trial (see Figure 4).

10. Repeat the procedure every day for a period of 10 days.

Note: After the 7th habituation trial, change the arrangement of the objects in one of the chamber zones in accordance with the experimental protocol for a given trial (see Figure 4).

\section{Data analysis}

1. Code behaviors on the basis of the recorded material using event logging software. This kind of software makes it possible to define particular behaviors and to score the time and frequency of selected behaviors.

2. Score selected behaviors occurring during the entire experimental session. The exact time of individual bouts of behavior, their frequency and, in consequence, the total time spent engaging in a given behavior, are assigned specific scores.

3. The measured behaviors comprise the following: total time spent in the unchanged zone of the chamber; total time spent in the changed zone of the chamber; time spent on contact with the tunnels (climbing the tunnels, entering inside the tunnels, touching with forelimbs, nose working in a distance less than $1 \mathrm{~cm}$ from the tunnel) in the unchanged zone of the chamber; frequency of contact with the tunnels in the unchanged zone of the chamber; total time spent on contact with the tunnels in the changed zone of the chamber; frequency of contact with the tunnels in the changed zone of the chamber; and frequency of moving between the chamber zones. Table 1 shows the ethogram prepared for BORIS coding software.

Table 1. Ethogram prepared for the purpose of the Pisula et al. (2019) study, to be used by BORIS software

\begin{tabular}{lll}
\hline Behavior code & Behavior type & Description \\
\hline Latency & State event ON/OFF & $\begin{array}{l}\text { The latency to leave the transporter } \\
\text { calculated from the moment a door to the } \\
\text { chamber are opened }\end{array}$
\end{tabular}


LeftZone

RightZone

InsideTransporter

LeftTunnelint

RightTunnelint

Grooming
State event ON/OFF

State event ON/OFF

State event ON/OFF

State event ON/OFF

State event ON/OFF

State event ON/OFF
Time spent in the left zone (at least head and forepaws in the zone)

Time spent in the right zone (at least head and forepaws in the zone)

Time spent in the transporter, whole body except tail

Time spent on contact with tunnels in a left zone

Time spent on contact with tunnels in a right zone

grooming

4. The experimental chamber design allows the experimenter to measure the time and frequency of object sniffing, upper sniffing, rearing, wall climbing, etc., which is done in order to analyze the specific exploratory behaviors.

5. To assess the emotional state of an animal, measure the latency to leave the transporter; the time and frequency of visits to the transporter, and the time and frequency of grooming. Grooming is considered a behavioral manifestation of the stress response measure in behavioral studies in rats (van Erp et al., 1994; Thor et al., 1988; Komorowska and Pisula, 2003).

6. Employ a multi-factor analysis with repeated measurements, followed by an LSD post-hoc test, with appropriate correction for multiple comparisons (e.g., Bonferroni).

\section{Notes}

1. Low-stress procedures require careful preparation of the experimental room in terms of sound, lighting and air-conditioning.

2. This protocol requires a habituation phase which is longer than the ones commonly used in other behavioral studies. Based on our experience from previous studies, we recommend that the habituation phase should be repeated each day for 7 days (one 7-min trial per day). The analysis of the behavior of many rat strains and laboratory short-tailed opossums, makes us confident that the animals achieve full familiarization with the test environment after completing this habituation plan.

3. During the test phase of the experiment, measure the activity of the animals in the zone where the configuration of objects has been modified. Repeat the test procedure at least three times. This enables a repeated measures analysis across both the habituation and the test trials. The standard plan of the experiment would therefore comprise seven habituation trials and three trials involving experimental manipulation. 
4. To avoid the confounding effect of lateralisation or visual/auditory cues, novelty should be introduced in the right chamber zone for half of the rats tested, and in the left chamber zone for the remaining half (e.g., a mirror image of the right part of Figure 4).

Important: If any animal shows an excessive right or left zone bias/preference during the habituation phase of the experiment, we strongly recommend excluding this individual from the successive trials.

5. This Protocol for measuring free (low-stress) exploration may be used in various forms and variants. Depending on the research question experimenters may prefer to use visual, olfactory or tactile characteristics of the novel stimulus. The experimental chamber may be easily adjusted to serve these purposes. The objects placed in the chamber zones may be manipulated, so as to decrease and increase environmental complexity or change the properties of the novel stimulus.

6. The experimental plan is based primarily on repeated measurements. This characteristic brings many benefits but sets several requirements as well. Firstly, it is essential to mark the low-stress individuals in order to code consecutive measurements correctly. Secondly, statistical methods need to be suitable for analyses involving within-subject effects (repeated measurements) and between-subject analyses (various manipulations).

\section{Acknowledgments}

The initial version of the protocol was applied in a study on RHA/RLA rats (Pisula, 2003). The latest version and most recent application have been described in a paper on Lister hooded rats (Pisula et al., 2019).

\section{Competing interests}

The authors declare no competing interests.

\section{Ethics}

Experimenters are advised to comply with the local ethical requirements regarding animal experimentation. It must be noted that this protocol itself does not involve any harmful or painful factor. Therefore in many countries, obtaining the ethical permission is not mandatory. However, the introduction of the stimulation, that may be stressful for animals, may lead to emergence of such necessity.

\section{References}

1. Calhoun, J. B. (1962). The ecology and sociology of the Norway rat. Bethesda: US Department 
of Health, Education, and Welfare.

2. Fanselow, M. S. and DeOca, B. M. (1998). Defensive Behaviors. In: Greenberg, G. and Haraway, M. (Eds.). Comparative psychology: A handbook (pp. 653-665). New York, Garland Publishers.

3. Friard, O. and Gamba, M. (2016). BORIS: a free, versatile open-source event-logging software for video/audio coding and live observations. Methods Ecol Evol 7(11): 1325-1330.

4. Gouteux, S., Vauclair, J. and Thinus-Blanc, C. (1999). Reaction to spatial novelty and exploratory strategies in baboons. Animal Learning Behavior 27(3): 323-332.

5. Komorowska, J. and Pisula, W. (2003). Does changing levels of stress affect the characteristics of grooming behavior in rats? Int J Com Psychol 16(4): 237246.

6. Picq, J. and Dhenain, J. (1998). Reaction to new objects and spatial changes in young and aged grey mouse lemurs (Microcebus murinus). The Quarterly J Exp Psychol 51B(4): 337-348.

7. Pisula, W. (2003). The Roman high- and low-avoidance rats respond differently to novelty in a familiarized environment. Behav Processes 63(2): 63-72.

8. Pisula, W., Modlinska, K. and Chrzanowska, A. (2019). Behavioural response to the environmental changes of various types in lister-hooded male rats. Sci Rep 9(1): 7111.

9. Renner, M. and Seltzer, C. (1991). Molar characteristics of exploratory and investigatory behavior in the rat (Rattus norvegicus). J Comp Psychol 105(4): 326-339.

10. Thor, D., Harrison, R., Schneider, R. and Carr, W. (1988). Sex differences in investigatory and grooming behaviors of laboratory rats (Rattus norvegicus) following exposure to novelty. J Comp Psychol 102(2): 188-192.

11. van Erp, A. M. M., Kruk, M. R., Meelis, W. and Willekens-Bramer, D. C. (1994). Effect of environmental stressors on time course, variability and form of self-grooming in the rat: Handling, social contact, defeat, novelty, restraint and fur moistening. Behav Brain Res 65(1): 47-55. 